PROCEEDINGS OF THE

AMERICAN MATHEMATICAL SOCIETY

Volume 139, Number 8, August 2011, Pages 2973-2986

S 0002-9939(2010)10716-5

Article electronically published on January 3, 2011

\title{
THE SCHRÖDINGER FOCK KERNEL AND THE NO-GO THEOREM FOR THE FIRST ORDER AND RENORMALIZED SQUARE OF WHITE NOISE LIE ALGEBRAS
}

\author{
LUIGI ACCARDI AND ANDREAS BOUKAS \\ (Communicated by Gail R. Letzter)
}

\begin{abstract}
Using the non-positive definiteness of the Fock kernel associated with the Schrödinger algebra we prove the impossibility of a joint Fock representation of the first order and Renormalized Square of White Noise Lie algebras with the convolution type renormalization $\delta^{2}(t-s)=\delta(s) \delta(t-s)$ for the square of the Dirac delta function. We show how the Schrödinger algebra Fock kernel can be reduced to a positive definite kernel through a restriction of the set of exponential vectors. We describe how the reduced Schrödinger kernel can be viewed as a tensor product of a Renormalized Square of White Noise $(s l(2))$ and a First Order of White Noise (Heisenberg) Fock kernel. We also compute the characteristic function of a stochastic process naturally associated with the reduced Schrödinger kernel.
\end{abstract}

\section{The RENORMALIZED HIGHER POWERS OF WHITE NOISE}

The quantum white noise functionals $b_{t}^{\dagger}$ (creation density) and $b_{t}$ (annihilation density) satisfy the Boson commutation relations

$$
\left[b_{t}, b_{s}^{\dagger}\right]=\delta(t-s),\left[b_{t}^{\dagger}, b_{s}^{\dagger}\right]=\left[b_{t}, b_{s}\right]=0,
$$

where $t, s \in \mathbb{R}$ and $\delta$ is the Dirac delta function, as well as the duality relation

$$
\left(b_{s}\right)^{*}=b_{s}^{\dagger} \text {. }
$$

In order to consider the smeared fields defined by the higher powers of $b_{t}$ and $b_{t}^{\dagger}$, for a test function $f$ and $n, k \in\{0,1,2, \ldots\}$, the sesquilinear forms

$$
B_{k}^{n}(f)=\int_{\mathbb{R}} f(t) b_{t}^{\dagger} b_{t}^{k} d t,
$$

where $d t$ denotes integration with respect to Lebesgue measure $\mu$, with involution

$$
\left(B_{k}^{n}(f)\right)^{*}=B_{n}^{k}(\bar{f})
$$

were defined in [7]. In [1] and [2] we introduced the convolution type renormalization

$$
\delta^{l}(t-s)=\delta(s) \delta(t-s), \quad l=2,3, \ldots
$$

Received by the editors April 5, 2010 and, in revised form, July 12, 2010 and July 22, 2010.

2010 Mathematics Subject Classification. Primary 60B15; Secondary 60H40, 17B45.

Key words and phrases. Schrödinger algebra, positive definite kernel, Fock representation, renormalized higher powers of white noise. 
of the higher powers of the Dirac delta function and, by restricting to test functions $f(t)$ such that $f(0)=0$, we obtained the Renormalized Higher Powers of White Noise $(R H P W N) *-$ Lie algebra commutation relations

$$
\left[B_{k}^{n}(f), B_{K}^{N}(g)\right]=(k N-K n) B_{k+K-1}^{n+N-1}(f g) .
$$

In particular, $B_{1}^{0}, B_{0}^{1}$ and $B_{0}^{0}$ satisfy the Heisenberg algebra (Heis) commutation relations

$$
\left[B_{1}^{0}(f), B_{0}^{1}(g)\right]=B_{0}^{0}(f g)
$$

and

$$
\left[B_{1}^{0}(f), B_{0}^{0}(g)\right]=\left[B_{0}^{1}(f), B_{0}^{0}(g)\right]=0,
$$

while $B_{2}^{0}, B_{0}^{2}$ and $B_{1}^{1}$ satisfy the Renormalized Square of White Noise $(R S W N)$ commutation relations

$$
\left[B_{2}^{0}(f), B_{0}^{2}(g)\right]=4 B_{1}^{1}(f g)
$$

and

$$
\left[B_{2}^{0}(f), B_{1}^{1}(g)\right]=2 B_{2}^{0}(f g),\left[B_{1}^{1}(f), B_{0}^{2}(g)\right]=2 B_{0}^{2}(f g),
$$

Notice that, for $I \subset \mathbb{R}$, the operators $R=\frac{1}{2} B_{0}^{2}\left(\chi_{I}\right), \Delta=\frac{1}{2} B_{2}^{0}\left(\chi_{I}\right)$ and $\rho=B_{1}^{1}\left(\chi_{I}\right)$ satisfy the $s l(2)$ commutation relations (see [8]). The classic form of the Heisenberg and $\operatorname{sl}(2)$ generators in terms of multiplication and differential operators can be found in [10].

\section{The Schrödinger Algebra In terms of the $R H P W N$ generators}

Let $I \subset \mathbb{R}$ with $\mu(I)>0$. In the notation of [9], the operators $M=B_{0}^{0}\left(\chi_{I}\right)=$ $\mu(I) I, K=2 B_{0}^{2}\left(\chi_{I}\right), G=2 B_{0}^{1}\left(\chi_{I}\right), D=B_{1}^{1}\left(\chi_{I}\right), P_{x}=\frac{1}{2} B_{1}^{0}\left(\chi_{I}\right)$, and $P_{t}=$ $\frac{1}{8} B_{2}^{0}\left(\chi_{I}\right)$ satisfy the commutation relations of the Schrödinger algebra given in the following table:

\begin{tabular}{c|cccccc} 
& $M$ & $K$ & $G$ & $D$ & $P_{x}$ & $P_{t}$ \\
\hline$M$ & 0 & 0 & 0 & 0 & 0 & 0 \\
$K$ & 0 & 0 & 0 & $-2 K$ & $-G$ & $-D$ \\
$G$ & 0 & 0 & 0 & $-G$ & $-M$ & $-P_{x}$ \\
$D$ & 0 & $2 K$ & $G$ & 0 & $-P_{x}$ & $-2 P_{t}$ \\
$P_{x}$ & 0 & $G$ & $M$ & $P_{x}$ & 0 & 0 \\
$P_{t}$ & 0 & $D$ & $P_{x}$ & $2 P_{t}$ & 0 & 0
\end{tabular}

The inner product (Leibniz function) of the exponential vectors

$$
\psi_{a, b}\left(\chi_{I}\right)=e^{a B_{0}^{2}\left(\chi_{I}\right)} e^{b B_{0}^{1}\left(\chi_{I}\right)} \Phi,
$$

where $a, b \in \mathbb{C}$ with $|a|<2$ and $\Phi$ is the Fock vacuum such that $B_{2}^{0}\left(\chi_{I}\right) \Phi=$ $B_{1}^{0}\left(\chi_{I}\right) \Phi=0$ and $B_{1}^{1}\left(\chi_{I}\right) \Phi=\frac{\mu(2)}{2} \Phi$, is given by (see Theorem 6.1 of [9] for $c=\mu(I) / 2$ and $a, b \in \mathbb{R}$; for a detailed proof see [10])

$$
\begin{aligned}
& \left\langle\psi_{a, b}\left(\chi_{I}\right), \psi_{A, B}\left(\chi_{I}\right)\right\rangle=\left(1-\frac{\bar{a} A}{4}\right)^{-\frac{\mu(I)}{2}} e^{\frac{\mu(I)}{4}\left(\frac{\bar{a} B^{2}+4 \bar{b} B+\bar{b}^{2} A}{4-\bar{a} A}\right)} \\
& =\exp \left(-\frac{\mu(I)}{2} \ln \left(1-\frac{\bar{a} A}{4}\right)\right) \exp \left(\frac{\mu(I)}{4}\left(\frac{\bar{a} B^{2}+4 \bar{b} B+\bar{b}^{2} A}{4-\bar{a} A}\right)\right) .
\end{aligned}
$$

Notice that for $a=A=0$ (2.3) reduces to the usual inner product of the Heisenberg algebra exponential vectors, and for $b=B=0$ it reduces to the inner product of 
the Square of White Noise Lie algebra (i.e. scaled $s l(2)$ ) exponential vectors (see [3]).

In [5] we showed that the Feinsilver-Kocik-Schott kernel (2.3) is not positive definite for arbitrary $\mu(I)$. That was done by showing that, in analogy with the no-go theorems of [1]- 3 ] and [6] for the impossibility of a Fock representation of the $R H P W N$ Lie algebra, for $\mu(I)$ below a certain threshold, "ghost" vectors (i.e. vectors of negative-norm) can be constructed. The existence of such a threshold is clarified in the following section.

To keep in connection with the origin of our work, i.e. the avoidance of the $R H P W N$ no-go theorems, in what follows we will use the notation $B_{1}^{0}, B_{0}^{1}, B_{0}^{0}$ and $B_{2}^{0}, B_{0}^{2}, B_{1}^{1}$ for the generators of a Heisenberg and an $\operatorname{sl}(2)$ (in $R S W N$ formulation) type Lie algebra respectively.

\section{The SCHRÖDINGER KERNEL AND THE NO-GO THEOREM FOR Heis AND RSWN}

As shown in $[9]$, for $\mu(I) \neq 0$ the operators

$$
\begin{aligned}
R_{0}\left(\chi_{I}\right): & =K-\frac{G^{2}}{2 m}=2 B_{0}^{2}\left(\chi_{I}\right)-\frac{2\left(B_{0}^{1}\left(\chi_{I}\right)\right)^{2}}{\mu(I)} \\
G\left(\chi_{I}\right): & =G=2 B_{0}^{1}\left(\chi_{I}\right)
\end{aligned}
$$

form an orthogonal basis for the Schrödinger "finite particle spaces", with

$$
\begin{gathered}
\left\langle R_{0}^{n}\left(\chi_{I}\right) G^{k}\left(\chi_{I}\right) \Phi, R_{0}^{N}\left(\chi_{I}\right) G^{K}\left(\chi_{I}\right) \Phi\right\rangle \\
=\left\langle\left(2 B_{0}^{2}\left(\chi_{I}\right)-\frac{2\left(B_{0}^{1}\left(\chi_{I}\right)\right)^{2}}{\mu(I)}\right)^{n}\left(2 B_{0}^{1}\left(\chi_{I}\right)\right)^{k} \Phi,\left(2 B_{0}^{2}\left(\chi_{I}\right)-\frac{2\left(B_{0}^{1}\left(\chi_{I}\right)\right)^{2}}{\mu(I)}\right)^{N}\left(2 B_{0}^{1}\left(\chi_{I}\right)\right)^{K} \Phi\right\rangle \\
=\delta_{n, N} \delta_{k, K} n ! k !\left(\frac{\mu(I)-1}{2}\right)_{n} \mu(I)^{k},
\end{gathered}
$$

where $\delta_{x, y}$ is Kronecker's delta and the increasing factorial powers $(x)_{y}$ are defined by $(x)_{y}:=x(x+1) \cdots(x+y-1)$, with $(x)_{0}=1$.

Notice that, for $\mu(I) \neq 0$,

$$
\begin{aligned}
B_{0}^{1}\left(\chi_{I}\right) & =\frac{1}{2} G\left(\chi_{I}\right), \\
B_{0}^{2}\left(\chi_{I}\right) & =\frac{1}{2} R_{0}\left(\chi_{I}\right)+\frac{1}{4 \mu(I)} G^{2}\left(\chi_{I}\right) .
\end{aligned}
$$

Passing to exponential vectors

$$
\phi_{a, b}\left(\chi_{I}\right):=e^{a R_{0}\left(\chi_{I}\right)} e^{b G\left(\chi_{I}\right)} \Phi=e^{a\left(2 B_{0}^{2}\left(\chi_{I}\right)-\frac{2\left(B_{0}^{1}\left(\chi_{I}\right)\right)^{2}}{\mu(I)}\right)} e^{b\left(2 B_{0}^{1}\left(\chi_{I}\right)\right)} \Phi
$$

where $a, b \in \mathbb{C}$, we have that

$$
\begin{aligned}
R_{0}\left(\chi_{I}\right) \phi_{a, b}\left(\chi_{I}\right) & =\frac{\partial}{\partial a} \phi_{a, b}\left(\chi_{I}\right)=\left.\frac{\partial}{\partial \epsilon}\right|_{\epsilon=0} \phi_{a+\epsilon, b}\left(\chi_{I}\right), \\
G\left(\chi_{I}\right) \phi_{a, b}\left(\chi_{I}\right) & =\frac{\partial}{\partial b} \phi_{a, b}\left(\chi_{I}\right)=\left.\frac{\partial}{\partial \epsilon}\right|_{\epsilon=0} \phi_{a, b+\epsilon}\left(\chi_{I}\right),
\end{aligned}
$$


and so

$$
\begin{aligned}
B_{0}^{1}\left(\chi_{I}\right) \phi_{a, b}\left(\chi_{I}\right) & =\frac{1}{2} \frac{\partial}{\partial b} \phi_{a, b}\left(\chi_{I}\right)=\left.\frac{1}{2} \frac{\partial}{\partial \epsilon}\right|_{\epsilon=0} \phi_{a, b+\epsilon}\left(\chi_{I}\right), \\
B_{0}^{2}\left(\chi_{I}\right) \phi_{a, b}\left(\chi_{I}\right) & =\left(\frac{1}{2} \frac{\partial}{\partial a}+\frac{1}{4 \mu(I)} \frac{\partial^{2}}{\partial b^{2}}\right) \phi_{a, b}\left(\chi_{I}\right) \\
& =\left.\frac{1}{2} \frac{\partial}{\partial \epsilon}\right|_{\epsilon=0} \phi_{a+\epsilon, b}\left(\chi_{I}\right)+\left.\frac{1}{4 \mu(I)} \frac{\partial^{2}}{\partial \epsilon^{2}}\right|_{\epsilon=0} \phi_{a, b+\epsilon}\left(\chi_{I}\right) .
\end{aligned}
$$

Theorem 3.1. For all $a, b, A, B \in \mathbb{C}$ and $I \subset \mathbb{R}$ with $\mu(I)>0$,

$$
\left\langle\phi_{a, b}\left(\chi_{I}\right), \phi_{A, B}\left(\chi_{I}\right)\right\rangle=(1-\bar{a} A)^{-\frac{\mu(I)-1}{2}} e^{\bar{b} B \mu(I)} .
$$

Proof.

$$
\begin{aligned}
& \left\langle\phi_{a, b}\left(\chi_{I}\right), \phi_{A, B}\left(\chi_{I}\right)\right\rangle \\
& =\sum_{n, k=0}^{\infty} \sum_{N, K=0}^{\infty} \frac{\bar{a}^{n} \bar{b}^{k} A^{N} B^{K}}{n ! k ! N ! K !} \delta_{n, N} \delta_{k, K} n ! k !\left(\frac{\mu(I)-1}{2}\right)_{n} \mu(I)^{k} \\
& =\sum_{n=0}^{\infty} \frac{(\bar{a} A)^{n}}{n !}\left(\frac{\mu(I)-1}{2}\right)_{n} \sum_{k=0}^{\infty} \frac{(\bar{b} B)^{k}}{k !} \mu(I)^{k} \\
& =(1-\bar{a} A)^{-\frac{\mu(I)-1}{2}} e^{\bar{b} B \mu(I)} .
\end{aligned}
$$

Corollary 1. The Schrödinger algebra Fock kernel (3.11) is positive definite if and only if $\mu(I) \geq 1$. Therefore we cannot jointly represent Heis and $R S W N$ on the same Fock space by insisting on including test functions defined on intervals $I$ of arbitrarily small measure $\mu(I)$.

Proof. By Schur's theorem, if $\frac{\mu(I)-1}{2} \geq 0$, i.e. if $\mu(I) \geq 1$, then the Schrödinger algebra Fock kernel (3.11) is positive definite as a product of a Heisenberg and an $s l(2)$ kernel. Conversely, if (3.11) is positive definite, then for $b=B=0$ we have that the kernel

$$
\left\langle\phi_{a, 0}\left(\chi_{I}\right), \phi_{A, 0}\left(\chi_{I}\right)\right\rangle=(1-\bar{a} A)^{-\frac{\mu(I)-1}{2}}
$$

is positive definite, which implies that $\mu(I) \geq 1$.

Remark. We could reduce (3.11) to a positive definite kernel through a restriction of the set of admissible test functions. Starting with exponential vectors of the form (3.6) with corresponding inner product (3.11), letting $\Lambda$ be an index set, we could define

$$
S_{\Lambda}:=\left\{I_{\lambda} \subset[0,+\infty), \lambda \in \Lambda / \mu\left(I_{\lambda}\right) \geq 1 \text { and } I_{\lambda} \cap I_{\lambda^{\prime}}=\emptyset \text { for } \lambda \neq \lambda^{\prime}\right\}
$$

and

$$
\mathcal{T}_{\Lambda}:=\left\{f=\sum_{i} a_{i} \chi_{I_{i}} / a_{i} \in \mathbb{C}, I_{i} \in S_{\Lambda}\right\} .
$$

For example, $\Lambda=\mathbb{N}_{0}$ and $S_{\mathbb{N}_{0}}=\left\{[n, n+1) / n \in \mathbb{N}_{0}\right\}$. If $f, g \in \mathcal{T}_{\Lambda}$ and $c \in \mathbb{C}$, then $f+c g, e^{c f}$ and $f g \in \mathcal{T}_{\Lambda}, 0 \in \mathcal{T}_{\Lambda}$. If we restrict to $S_{\Lambda}$ and $\mathcal{T}_{\Lambda}$, then both (3.11) and (5.4) are positive definite and we have a Fock representation of the second quantized Schrödinger algebra. The second quantized Schrödinger Fock space would then be defined as the tensor product $\otimes_{\lambda}$ of the one-mode, i.e. restricted to a single interval, Schrödinger Fock spaces over the $I_{\lambda}$ 's. 


\section{Adjoint action on the exponential vectors}

Theorem 4.1. The adjoints of $R_{0}\left(\chi_{I}\right), G\left(\chi_{I}\right), B_{0}^{2}\left(\chi_{I}\right)$ and $B_{0}^{1}\left(\chi_{I}\right)$ are given by

$$
\begin{aligned}
\left(R_{0}\right)^{*}\left(\chi_{I}\right) \phi_{a, b}\left(\chi_{I}\right) & =\left(\frac{\mu(I)-1}{2} a+a^{2} \frac{\partial}{\partial a}\right) \phi_{a, b}\left(\chi_{I}\right) \\
& =\frac{\mu(I)-1}{2} a \phi_{a, b}\left(\chi_{I}\right)+\left.a^{2} \frac{\partial}{\partial \epsilon}\right|_{\epsilon=0} \phi_{a+\epsilon, b}\left(\chi_{I}\right) \\
G^{*}\left(\chi_{I}\right) \phi_{a, b}\left(\chi_{I}\right) & =\mu(I) b \phi_{a, b}\left(\chi_{I}\right), \\
B_{2}^{0}\left(\chi_{I}\right) \phi_{a, b}\left(\chi_{I}\right) & =\left(\frac{\mu(I)-1}{4} a+\frac{a^{2}}{2} \frac{\partial}{\partial a}+\frac{\mu(I)}{4} b^{2}\right) \phi_{a, b}\left(\chi_{I}\right) \\
& =\left(\frac{\mu(I)-1}{4} a+\frac{\mu(I)}{4} b^{2}\right) \phi_{a, b}\left(\chi_{I}\right)+\left.\frac{a^{2}}{2} \frac{\partial}{\partial \epsilon}\right|_{\epsilon=0} \phi_{a+\epsilon, b}\left(\chi_{I}\right),
\end{aligned}
$$

and

$$
B_{1}^{0}\left(\chi_{I}\right) \phi_{a, b}\left(\chi_{I}\right)=\frac{\mu(I)}{2} b \phi_{a, b}\left(\chi_{I}\right) .
$$

Proof. The proof follows by combining the fact that (3.11) is split into an $s l(2)$ and

a Heisenberg part and the results of section 4 of [10] (with $c=\frac{\mu(I)-1}{2}$ ) on the form of annihilation (lowering) and creation (raising) operators in the two algebras. A direct proof can be given by showing that, for example,

$$
\begin{aligned}
\left\langle\left(R_{0}\right)^{*}\left(\chi_{I}\right) \phi_{a, b}\left(\chi_{I}\right), \phi_{A, B}\left(\chi_{I}\right)\right\rangle & =\left(\frac{\mu(I)-1}{2} \bar{a}+\bar{a}^{2} \frac{\partial}{\partial \bar{a}}\right)(1-\bar{a} A)^{-\frac{\mu(I)-1}{2}} e^{\bar{b} B \mu(I)} \\
& =\frac{\partial}{\partial A}(1-\bar{a} A)^{-\frac{\mu(I)-1}{2}} e^{\bar{b} B \mu(I)} \\
& =\left\langle\phi_{a, b}\left(\chi_{I}\right), R_{0}\left(\chi_{I}\right) \phi_{A, B}\left(\chi_{I}\right)\right\rangle .
\end{aligned}
$$

\section{EXTENSION TO STEP FUNCTIONS}

Using the commutativity of the Schrödinger algebra generators on disjoint sets, we may extend the kernel (2.3) to exponential vectors of the form

$$
\Psi(f, g):=\prod_{i} e^{a_{i} B_{0}^{2}\left(\chi_{I_{i}}\right)} e^{b_{i} B_{0}^{1}\left(\chi_{I_{i}}\right)} \Phi=e^{B_{0}^{2}(f)} e^{B_{0}^{1}(g)} \Phi,
$$

where $f=\sum_{i} a_{i} \chi_{I_{i}}$ and $g=\sum_{i} b_{i} \chi_{I_{i}}$, with $I_{i} \cap I_{j}=\oslash$ for $i \neq j$, are simple functions with $|f|<2$, and we obtain

$$
\begin{aligned}
\left\langle\Psi\left(f_{1}, g_{1}\right), \Psi\left(f_{2}, g_{2}\right)\right\rangle= & \exp \left(-\frac{1}{2} \int \ln \left(1-\frac{\bar{f}_{1} f_{2}}{4}\right) d \mu\right) \\
& \times \exp \left(\frac{1}{4} \int\left(\frac{\bar{f}_{1} g_{2}^{2}+4 \bar{g}_{1} g_{2}+\bar{g}_{1}^{2} f_{2}}{4-\bar{f}_{1} f_{2}}\right) d \mu\right) .
\end{aligned}
$$


We may extend (3.6) and (3.11) to step functions $f=\sum_{i} a_{i} \chi_{I_{i}}$ and $g=\sum_{i} b_{i} \chi_{I_{i}}$, with $|f|<1$ and $I_{i} \cap I_{j}=\oslash$ for $i \neq j$, through

$$
\begin{aligned}
\phi(f, g) & =e^{R_{0}(f)} e^{G(g)} \Phi=\prod_{i} e^{a_{i} R_{0}\left(\chi_{I_{i}}\right)} e^{b_{i} G\left(\chi_{I_{i}}\right)} \Phi \\
& =\prod_{i} e^{a_{i}\left(2 B_{0}^{2}\left(\chi_{I_{i}}\right)-\frac{2\left(B_{0}^{1}\left(\chi_{I_{i}}\right)^{2}\right.}{\mu\left(I_{i}\right)}\right)} e^{b_{i}\left(2 B_{0}^{1}\left(\chi_{I_{i}}\right)\right)} \Phi,
\end{aligned}
$$

and for $f_{1}=\sum_{i} a_{i} \chi_{I_{i}}, f_{2}=\sum_{i} A_{i} \chi_{I_{i}}, g_{1}=\sum_{i} b_{i} \chi_{I_{i}}$ and $g_{2}=\sum_{i} B_{i} \chi_{I_{i}}$,

$$
\begin{aligned}
& \left\langle\phi\left(f_{1}, g_{1}\right), \phi\left(f_{2}, g_{2}\right)\right\rangle=\prod_{i}\left(1-\bar{a}_{i} A_{i}\right)^{-\frac{\mu\left(I_{i}\right)-1}{2}} e^{\bar{b}_{i} B_{i} \mu\left(I_{i}\right)} \\
= & \pi\left(f_{1}, f_{2}\right) \exp \left(-\frac{1}{2} \int \ln \left(1-\bar{f}_{1} f_{2}\right) d \mu\right) \exp \left(\int \bar{g}_{1} g_{2} d \mu\right)
\end{aligned}
$$

respectively, where

$$
\pi\left(f_{1}, f_{2}\right):=\prod_{i}\left(1-\overline{a_{i}} A_{i}\right)^{1 / 2} .
$$

By Schur's Lemma, (5.4) is a positive definite kernel provided that $\mu\left(I_{i}\right) \geq 1$ for all $i$. Moreover, for step functions $f, g, h$,

$$
\begin{aligned}
R_{0}(h) \phi(f, g) & =\left.\frac{\partial}{\partial \epsilon}\right|_{\epsilon=0} \phi(f+\epsilon h, g), \\
G(h) \phi(f, g) & =\left.\frac{\partial}{\partial \epsilon}\right|_{\epsilon=0} \phi(f, g+\epsilon h),
\end{aligned}
$$

and so

$$
B_{0}^{1}(h) \phi(f, g)=\left.\frac{1}{2} \frac{\partial}{\partial \epsilon}\right|_{\epsilon=0} \phi(f, g+\epsilon h)
$$

and

$$
\begin{aligned}
B_{0}^{2}(h) \phi(f, g)= & \left.\frac{1}{2} \frac{\partial}{\partial \epsilon}\right|_{\epsilon=0} \phi(f+\epsilon h, g) \\
& +\left.\frac{1}{4} \frac{\partial^{2}}{\partial \epsilon^{2}}\right|_{\epsilon=0} \phi(f, g+\epsilon \hat{h}) \quad(\text { for } h>0),
\end{aligned}
$$

where for $h=\sum_{i} h_{i} \chi_{I_{i}}$ we define $\hat{h}=\sum_{i} \hat{h}_{i} \chi_{I_{i}}$, where $\hat{h}_{i}=\left(\frac{h_{i}}{\mu\left(I_{i}\right)}\right)^{1 / 2}$.

The adjoint action of $B_{0}^{1}(h)$ and $B_{0}^{2}(h)$ on the exponential vectors $\phi(f, g)$ is given by

$$
B_{1}^{0}(h) \phi(f, g)=\frac{1}{2} \int h g d \mu \phi(f, g)
$$

and

$$
\begin{aligned}
B_{2}^{0}(h) \phi(f, g)= & \frac{1}{4}\left(\int h\left(f+g^{2}\right) d \mu-\operatorname{Tr}(h f)\right) \phi(f, g) \\
& +\left.\frac{1}{2} \frac{\partial}{\partial \epsilon}\right|_{\epsilon=0} \phi\left(f+\epsilon h f^{2}, g\right),
\end{aligned}
$$

where for $h=\sum_{i} h_{i} \chi_{I_{i}}$ and $g=\sum_{i} g_{i} \chi_{I_{i}}$ we define $\operatorname{Tr}(h f)=\sum_{i} h_{i} g_{i}$.

As in Theorem 4.1, we may verify (5.10)-(5.11) directly by checking that, for each pair $\left(X, X^{*}\right)=\left(B_{0}^{1}(h), B_{1}^{0}(\bar{h})\right)$ and $\left(X, X^{*}\right)=\left(B_{0}^{2}(h), B_{2}^{0}(\bar{h})\right)$,

$$
\left\langle X \phi\left(f_{1}, g_{1}\right), \phi\left(f_{2}, g_{2}\right)\right\rangle=\left\langle\phi\left(f_{1}, g_{1}\right), X^{*} \phi\left(f_{2}, g_{2}\right)\right\rangle \text {. }
$$




\section{Positive definite Restrictions of the Schrödinger Kernel}

Investigating the non-positive definiteness of the kernel (2.3), we look for a restriction of the parameters $a, b \in \mathbb{C},|a|<2$, in the definition (2.2) of the exponential vectors $\psi_{a, b}\left(\chi_{I}\right)$ that will result in a positive definite kernel. We notice that if $a, b, A, B \in \mathbb{C}$ are such that

$$
\bar{a} B^{2}+\bar{b}^{2} A=0,
$$

i.e. if $(a, b),(A, B) \in S_{\lambda}$ where, for given $\lambda \in \mathbb{R}$,

$$
S_{\lambda}=\left\{(a, b) \in \mathbb{C}^{2} / a=i \lambda b^{2},|\lambda||b|^{2}<2\right\},
$$

then the kernel (2.3) reduces to

$$
\begin{aligned}
\left\langle\psi_{a, b}\left(\chi_{I}\right), \psi_{A, B}\left(\chi_{I}\right)\right\rangle & =\left(1-\frac{\bar{a} A}{4}\right)^{-\frac{\mu(I)}{2}} \exp \left(\frac{\mu(I)}{4} \frac{\bar{b} B}{1-\frac{\bar{a} A}{4}}\right) \\
& =\left(1-\frac{\bar{a} A}{4}\right)^{-\frac{\mu(I)}{2}} \exp \left(\frac{\mu(I)}{4}(\bar{b} B) \sum_{n=0}^{\infty}\left(\frac{\bar{a} A}{4}\right)^{n}\right) .
\end{aligned}
$$

The kernels $K_{i}: \mathbb{C}^{2} \times \mathbb{C}^{2} \rightarrow \mathbb{C}$ defined for $i \in\{1,2,3\}$ by

$$
\begin{aligned}
K_{1}((a, b),(A, B)) & =\left(1-\frac{\bar{a} A}{4}\right)^{-\frac{\mu(I)}{2}}, \\
K_{2}((a, b),(A, B)) & =\frac{\mu(I)}{4} \bar{b} B \\
K_{3}((a, b),(A, B)) & =\lim _{\rho \rightarrow \infty} K_{3, \rho}((a, b),(A, B))
\end{aligned}
$$

where, for $\rho \geq 1$,

$$
K_{3, \rho}((a, b),(A, B))=\sum_{n=0}^{\rho}\left(\frac{\bar{a} A}{4}\right)^{n}
$$

are positive definite, since $K_{1}$ is the well-known $s l(2)$ kernel, $K_{2}$ and $K_{3, \rho}$ are Heisenberg-type kernels and $K_{3}$ is a limit of positive definite kernels. Therefore $K_{1} e^{K_{2} K_{3}}$, i.e. (6.3), is also a positive definite kernel. Notice that a direct proof of the positive definiteness of $K_{1}$ can be given by using the power series expansion

$$
-\ln (1-z)=\sum_{n=1}^{\infty} \frac{z^{n}}{n}
$$

valid for $|z|<1$.

From a geometric point of view, letting $a=X+i Y$ and $b=x+i y$, the defining relation $a=i \lambda b^{2}$ of $S_{\lambda}$ corresponds to the hyperbolic paraboloids

$$
\begin{aligned}
X & =-2 \lambda x y, \\
Y & =\lambda\left(x^{2}-y^{2}\right) .
\end{aligned}
$$

For given $\lambda \in \mathbb{R}$ and $I \subset \mathbb{R}$ we will use the notation

$$
\psi(b)=\psi_{i \lambda b^{2}, b}\left(\chi_{I}\right)=e^{i \lambda b^{2} B_{0}^{2}\left(\chi_{I}\right)} e^{b B_{0}^{1}\left(\chi_{I}\right)} \Phi
$$


with inner product

$$
\begin{aligned}
\langle\psi(b), \psi(B)\rangle & =\left\langle\psi_{i \lambda b^{2}, b}\left(\chi_{I}\right), \psi_{i \lambda B^{2}, B}\left(\chi_{I}\right)\right\rangle \\
& =\left(1-\frac{\lambda^{2}}{4}(\bar{b} B)^{2}\right)^{-\nu(I)} \exp \left(\frac{\bar{b} B}{4-\lambda^{2}(\bar{b} B)^{2}} \mu(I)\right)
\end{aligned}
$$

where

$$
\nu=\frac{\mu}{2}
$$

Definition 6.1. For $\lambda \in \mathbb{R}$ we define the (restricted) Schrödinger Fock space $\mathcal{F}_{\mathcal{S}}(\lambda)$ as the closure of the linear span of the exponential vectors $\psi(b)$ defined in (6.11) with respect to the inner product (6.12).

We notice that $B_{0}^{2}\left(\chi_{I}\right)$ and $B_{0}^{1}\left(\chi_{I}\right)$ cannot be separately defined on $\mathcal{F}_{\mathcal{S}}(\lambda)$, since that would require a change in $\lambda$. Specifically, for $b \neq 0$

$$
\begin{aligned}
B_{0}^{2}\left(\chi_{I}\right) \psi(b) & =\frac{1}{i b^{2}} \frac{\partial}{\partial \lambda} \psi(b) \\
B_{0}^{1}\left(\chi_{I}\right) \psi(b) & =\left(\frac{\partial}{\partial b}-\frac{2 \lambda}{b} \frac{\partial}{\partial \lambda}\right) \psi(b) .
\end{aligned}
$$

\section{7. $\mathcal{F}_{\mathcal{S}}(\lambda)$ AS A TENSOR PRODUCT}

In what follows, we fix $I \subset \mathbb{R}$ with $\mu(I)>0$.

Definition 7.1. For $\lambda \in \mathbb{R} \backslash\{0\}$ and $b \in \mathbb{C}$, we denote by $\Phi_{R S W N}$ the vacuum vector and by

$$
\epsilon(b)=\exp \left(\frac{\lambda b^{2}}{4} B_{0}^{2}\left(\chi_{I}\right)\right) \Phi_{R S W N}
$$

the exponential vectors of the Fock space $\mathcal{F}_{R S W N}$ defined as the completion of the linear span of the exponential vectors (7.1) with respect to the inner product

$$
\langle\epsilon(b), \epsilon(B)\rangle=\exp \left(-\ln \left(1-\frac{\lambda^{2}(\bar{b} B)^{2}}{4}\right) \nu(I)\right)=\left(1-\frac{\lambda^{2}(\bar{b} B)^{2}}{4}\right)^{-\nu(I)} .
$$

Theorem 7.2. On the exponential domain of $\mathcal{F}_{R S W N}$, i.e. on the linear span of the $\epsilon(b)$ 's:

(i) the adjoint $B_{2}^{0}\left(\chi_{I}\right)$ of the operator $B_{0}^{2}\left(\chi_{I}\right)$ defined by

$$
B_{0}^{2}\left(\chi_{I}\right) \epsilon(b)=\frac{2}{\lambda b} \frac{\partial}{\partial b} \epsilon(b) \quad(b \neq 0)
$$

is given by

$$
B_{2}^{0}\left(\chi_{I}\right) \epsilon(b)=\left(\lambda b^{2} \nu(I)+\frac{\lambda b^{3}}{2} \frac{\partial}{\partial b}\right) \epsilon(b)
$$

(ii) the operators $B_{2}^{0}\left(\chi_{I}\right), B_{0}^{2}\left(\chi_{I}\right)$ and $B_{1}^{1}\left(\chi_{I}\right)$ defined by

$$
B_{1}^{1}\left(\chi_{I}\right) \epsilon(b)=\left(\nu(I)+b \frac{\partial}{\partial b}\right) \epsilon(b)
$$

satisfy the RSWN commutation relations (1.9) and (1.10). 
Proof. To prove (7.4) we notice that for all $b, B \in \mathbb{C}$

$$
\begin{aligned}
\left\langle B_{0}^{2}\left(\chi_{I}\right) \epsilon(b), \epsilon(B)\right\rangle & =\left\langle\frac{2}{\lambda b} \frac{\partial}{\partial b} \epsilon(b), \epsilon(B)\right\rangle \\
& =\frac{2}{\lambda \bar{b}} \frac{\partial}{\partial \bar{b}}\langle\epsilon(b), \epsilon(B)\rangle \\
& =\frac{2}{\lambda \bar{b}} \frac{\partial}{\partial \bar{b}}\left(1-\frac{\lambda^{2}(\bar{b} B)^{2}}{4}\right)^{-\nu(I)} \\
& =\frac{\lambda B^{2} \nu(I)}{1-\frac{\lambda^{2}(\bar{b} B)^{2}}{4}}\left(1-\frac{\lambda^{2}(\bar{b} B)^{2}}{4}\right)^{-\nu(I)} \\
& =\left(\lambda B^{2} \nu(I)+\frac{\lambda B^{3}}{2} \frac{\partial}{\partial B}\right)\left(1-\frac{\lambda^{2}(\bar{b} B)^{2}}{4}\right)^{-\nu(I)} \\
& =\left\langle\epsilon(b), B_{2}^{0}\left(\chi_{I}\right) \epsilon(B)\right\rangle .
\end{aligned}
$$

To prove (1.9) we notice that

$$
\begin{gathered}
\left\langle\epsilon(b),\left[B_{2}^{0}\left(\chi_{I}\right), B_{0}^{2}\left(\chi_{I}\right)\right] \epsilon(B)\right\rangle \\
=\left\langle B_{0}^{2}\left(\chi_{I}\right) \epsilon(b), B_{0}^{2}\left(\chi_{I}\right) \epsilon(B)\right\rangle-\left\langle B_{2}^{0}\left(\chi_{I}\right) \epsilon(b), B_{2}^{0}\left(\chi_{I}\right) \epsilon(B)\right\rangle \\
=\left(\left(\frac{2}{\lambda \bar{b}} \frac{\partial}{\partial \bar{b}}\right)\left(\frac{2}{\lambda B} \frac{\partial}{\partial B}\right)-\left(\lambda \bar{b}^{2} \nu(I)+\frac{\lambda \bar{b}^{3}}{2} \frac{\partial}{\partial \bar{b}}\right)\left(\lambda B^{2} \nu(I)+\frac{\lambda B^{3}}{2} \frac{\partial}{\partial B}\right)\right)\langle\epsilon(b), \epsilon(B)\rangle \\
=\frac{4+\lambda^{2}(\bar{b} B)^{2}}{1-\frac{\lambda^{2}(\bar{b} B)^{2}}{4}} \nu(I)\left(1-\frac{\lambda^{2}(\bar{b} B)^{2}}{4}\right)^{-\nu(I)} \\
=4\left(\nu(I)+B \frac{\partial}{\partial B}\right)\langle\epsilon(b), \epsilon(B)\rangle \\
=\left\langle\epsilon(b), 4 B_{1}^{1}\left(\chi_{I}\right) \epsilon(B)\right\rangle .
\end{gathered}
$$

The proof of (1.10) is similar.

Notice that, in agreement with $[\underline{3}$,

$$
B_{2}^{0}\left(\chi_{I}\right) \Phi_{R S W N}=0 ; B_{1}^{1}\left(\chi_{I}\right) \Phi_{R S W N}=\frac{\mu(I)}{2} \Phi_{R S W N} .
$$

Lemma 7.3. For all operators $A$ and $B$ acting on vector spaces $V$ and $W$ respectively, assuming that all symbols are well-defined,

$$
e^{A \otimes I+I \otimes B}=e^{A} \otimes e^{B},
$$

where I denotes the identity operator in the respective space. 
Proof. Since $A \otimes I$ and $I \otimes B$ commute,

$$
\begin{aligned}
e^{A \otimes I+I \otimes B} & =e^{A \otimes I} e^{I \otimes B} \\
& =\left(\sum_{n=0}^{\infty} \frac{1}{n !}(A \otimes I)^{n}\right)\left(\sum_{k=0}^{\infty} \frac{1}{k !}(I \otimes B)^{k}\right) \\
& =\left(\sum_{n=0}^{\infty} \frac{1}{n !}\left(A^{n} \otimes I\right)\right)\left(\sum_{k=0}^{\infty} \frac{1}{k !}\left(I \otimes B^{k}\right)\right) \\
& =\sum_{n, k=0}^{\infty} \frac{1}{n ! k !}\left(A^{n} \otimes B^{k}\right) \\
& =\left(\sum_{n=0}^{\infty} \frac{1}{n !} A^{n}\right) \otimes\left(\sum_{k=0}^{\infty} \frac{1}{k !} B^{k}\right) \\
& =e^{A} \otimes e^{B} .
\end{aligned}
$$

Assuming convergence, we may extend (7.7) to infinite sums of operators of the form $I_{0} \otimes \cdots \otimes I_{k-1} \otimes A_{k}(t) \otimes I_{k+1} \otimes \cdots$ in the sense that

$$
\exp \left(\sum_{k=0}^{\infty}\left(I_{0} \otimes \cdots \otimes I_{k-1} \otimes A_{k}(t) \otimes I_{k+1} \otimes \cdots\right)\right)=\bigotimes_{k=0}^{\infty} \exp \left(A_{k}(t)\right) .
$$

Definition 7.4. For $\lambda \in \mathbb{R}, n \geq 0$ and $b \in \mathbb{C}$, we denote by $\Phi_{n}$ the vacuum vector and by

$$
\epsilon_{n}(b)=\exp \left(b^{2 n+1} B_{0}^{1}\left(\chi_{I} ; n\right)\right) \Phi_{n}
$$

the exponential vectors of the Fock space $\mathcal{F}_{H_{n}}$ defined as the completion of the linear span of the exponential vectors (7.9) with respect to the inner product

$$
\left\langle\epsilon_{n}(b), \epsilon_{n}(B)\right\rangle=\exp \left((\bar{b} B)^{2 n+1} \mu_{n}(I)\right),
$$

where $\mu_{n}=\frac{1}{4}\left(\frac{\lambda}{2}\right)^{2 n} \mu$.

Theorem 7.5. In the notation of Definition 7.4, on the exponential domain of $\mathcal{F}_{H_{n}}$, i.e. on the linear span of the $\epsilon_{n}(b)$ 's:

(i) the adjoint of the operator $B_{0}^{1}\left(\chi_{I} ; n\right)$ defined by

$$
B_{0}^{1}\left(\chi_{I} ; n\right) \epsilon_{n}(b)=\frac{1}{(2 n+1) b^{2 n}} \frac{\partial}{\partial b} \epsilon_{n}(b) \quad(b \neq 0)
$$

is the operator $B_{1}^{0}\left(\chi_{I} ; n\right)$ defined by

$$
B_{1}^{0}\left(\chi_{I} ; n\right) \epsilon_{n}(b)=b^{2 n+1} \mu_{n}(I) \epsilon_{n}(b) ;
$$

(ii) the operators $B_{0}^{1}\left(\chi_{I} ; n\right), B_{1}^{0}\left(\chi_{I} ; n\right)$ and $B_{0}^{0}\left(\chi_{I} ; n\right)$ defined by

$$
B_{0}^{0}\left(\chi_{I} ; n\right) \epsilon_{n}(b)=\mu_{n}(I) \epsilon_{n}(b)
$$

satisfy the First Order of White Noise (i.e. Heisenberg) commutation relations (1.7) and (1.8) as well as the involution condition (1.4).

Proof. The proof is similar to that of Theorem 7.2 . 
Definition 7.6. For $|\lambda|<2$ (to ensure the weak convergence of all infinite sums and tensor products appearing in what follows), we denote by $\Phi_{H}=\bigotimes_{n=0}^{\infty} \Phi_{n}$ the vacuum vector and, for $b \in \mathbb{C}$, by

$$
=\exp \left(\sum_{n=0}^{\infty}\left(I_{\mathcal{F}_{H_{0}}} \otimes \cdots \otimes I_{\mathcal{F}_{H_{n-1}}} \otimes b^{2 n+1} B_{0}^{1}\left(\chi_{I} ; n\right) \otimes I_{\mathcal{F}_{H_{n+1}}} \otimes \cdots\right)\right) \Phi_{H}
$$

the exponential vectors of the Fock space

$$
\mathcal{F}_{H}=\bigotimes_{n=0}^{\infty} \mathcal{F}_{H_{n}}
$$

defined as the completion of the linear span of the exponential vectors (7.14) with respect to the inner product

$$
\langle\eta(b), \eta(B)\rangle=\prod_{n=0}^{\infty}\left\langle\epsilon_{n}(b), \epsilon_{n}(B)\right\rangle=\exp \left(\frac{\bar{b} B}{4-\lambda^{2}(\bar{b} B)^{2}} \mu(I)\right)
$$

Theorem 7.7. In the notation of Definition 7.6, on the exponential domain, i.e. on the linear span of the $\eta(b)$ 's:

(i) the adjoint of the operator $B_{0}^{1}\left(\chi_{I}\right)$ defined by

$$
\begin{gathered}
B_{0}^{1}\left(\chi_{I}\right) \eta(b) \\
=\sum_{n=0}^{\infty}\left(I_{\mathcal{F}_{H_{0}}} \otimes \cdots \otimes I_{\mathcal{F}_{H_{n-1}}} \otimes B_{0}^{1}\left(\chi_{I} ; n\right) \otimes I_{\mathcal{F}_{H_{n+1}}} \otimes \cdots\right) \eta(b) \\
=\sum_{n=0}^{\infty}\left(I_{\mathcal{F}_{H_{0}}} \otimes \cdots \otimes I_{\mathcal{F}_{H_{n-1}}} \otimes \frac{1}{(2 n+1) b^{2 n}} \frac{\partial}{\partial b} \otimes I_{\mathcal{F}_{H_{n+1}}} \otimes \cdots\right) \eta(b)
\end{gathered}
$$

is the operator $B_{1}^{0}\left(\chi_{I}\right)$ defined by

$$
\begin{gathered}
B_{1}^{0}\left(\chi_{I}\right) \eta(b) \\
=\sum_{n=0}^{\infty}\left(I_{\mathcal{F}_{H_{0}}} \otimes \cdots \otimes I_{\mathcal{F}_{H_{n-1}}} \otimes B_{1}^{0}\left(\chi_{I} ; n\right) \otimes I_{\mathcal{F}_{H_{n+1}}} \otimes \cdots\right) \eta(b) \\
=\sum_{n=0}^{\infty}\left(I_{\mathcal{F}_{H_{0}}} \otimes \cdots \otimes I_{\mathcal{F}_{H_{n-1}}} \otimes b^{2 n+1} \mu_{n}(I) \otimes I_{\mathcal{F}_{H_{n+1}}} \otimes \cdots\right) \eta(b) ;
\end{gathered}
$$

(ii) the operators $B_{0}^{1}\left(\chi_{I}\right), B_{1}^{0}\left(\chi_{I}\right)$ and $B_{0}^{0}\left(\chi_{I}\right)$ defined by

$$
B_{0}^{0}\left(\chi_{I}\right) \eta(b)=\sum_{n=0}^{\infty}\left(I_{\mathcal{F}_{H_{0}}} \otimes \cdots \otimes I_{\mathcal{F}_{H_{n-1}}} \otimes \mu_{n}(I) \otimes I_{\mathcal{F}_{H_{n+1}}} \otimes \cdots\right) \eta(b)
$$

satisfy the First Order of White Noise (Heisenberg) commutation relations (1.7) and (1.8) as well as the involution condition (1.4). 
Proof. The proof of (i) follows by applying Theorem 7.2 to the $n$-th factor space. To prove (ii) we notice that, weakly on the exponential domain

$$
\begin{gathered}
{\left[B_{1}^{0}\left(\chi_{I}\right), B_{0}^{1}\left(\chi_{I}\right)\right]} \\
=\left[\sum_{n=0}^{\infty}\left(I_{\mathcal{F}_{H_{0}}} \otimes \cdots \otimes I_{\mathcal{F}_{H_{n-1}}} \otimes B_{1}^{0}\left(\chi_{I} ; n\right) \otimes I_{\mathcal{F}_{H_{n+1}}} \otimes \cdots\right),\right. \\
\left.\sum_{N=0}^{\infty}\left(I_{\mathcal{F}_{H_{0}}} \otimes \cdots \otimes I_{\mathcal{F}_{H_{N-1}}} \otimes B_{0}^{1}\left(\chi_{I} ; N\right) \otimes I_{\mathcal{F}_{H_{N+1}}} \otimes \cdots\right)\right] \\
=\sum_{n=0}^{\infty}\left[\left(I_{\mathcal{F}_{H_{0}}} \otimes \cdots \otimes I_{\mathcal{F}_{H_{n-1}}} \otimes B_{1}^{0}\left(\chi_{I} ; n\right) \otimes I_{\mathcal{F}_{H_{n+1}}} \otimes \cdots\right),\right. \\
\left.\left(I_{\mathcal{F}_{H_{0}}} \otimes \cdots \otimes I_{\mathcal{F}_{H_{n-1}}} \otimes B_{0}^{1}\left(\chi_{I} ; n\right) \otimes I_{\mathcal{F}_{H_{n+1}}} \otimes \cdots\right)\right] \\
=\sum_{n=0}^{\infty}\left(I_{\mathcal{F}_{H_{0}}} \otimes \cdots \otimes I_{\mathcal{F}_{H_{n-1}}} \otimes\left[B_{1}^{0}\left(\chi_{I} ; n\right), B_{0}^{1}\left(\chi_{I} ; n\right)\right] \otimes I_{\mathcal{F}_{H_{n+1}}} \otimes \cdots\right) \\
=\sum_{n=0}^{\infty}\left(I_{\mathcal{F}_{H_{0}}} \otimes \cdots \otimes I_{\mathcal{F}_{H_{n-1}}} \otimes \mu_{n}(I) \otimes I_{\mathcal{F}_{H_{n+1}}} \otimes \cdots\right) \\
=B_{0}^{0}\left(\chi_{I}\right) .
\end{gathered}
$$

Theorem 7.8. For $0<|\lambda|<2$

$$
\mathcal{F}_{\lambda}=\mathcal{F}_{R S W N} \otimes \mathcal{F}_{H}
$$

and

$$
\psi(b)=\epsilon(b) \otimes \eta(b) .
$$

Proof. The proof follows from the fact that

$$
\langle\psi(b), \psi(B)\rangle=\langle\epsilon(b), \epsilon(B)\rangle\langle\eta(b), \eta(B)\rangle .
$$

\section{Stochastic processes on $\mathcal{F}_{\mathcal{S}}(\lambda)$}

From a probabilistic point of view, tensor products of Fock spaces correspond to independent random variables associated with mutually commuting Lie algebras (see [8]).

For $t \geq 0$, let $C(t)=B_{0}^{2}\left(\chi_{[0, t]}\right)+B_{2}^{0}\left(\chi_{[0, t]}\right)$ and

$$
X_{n}(t)=B_{0}^{1}\left(\chi_{[0, t]} ; n\right)+B_{1}^{0}\left(\chi_{[0, t]} ; n\right),
$$

where $C(t)$ is defined by (7.3) and (7.4) and, for each $n \geq 0, X_{n}(t)$ is defined by (7.11) and (7.12). Define

$$
X(t)=\hat{C}(t)+\sum_{n=0}^{\infty} \hat{X}_{n}(t)
$$

where

$$
\begin{gathered}
\hat{C}(t)=C(t) \otimes I_{\mathcal{F}_{H}}, \\
\hat{X}_{0}(t)=I_{\mathcal{F}_{R S W N}} \otimes I_{\mathcal{F}_{H_{0}}} \otimes I_{\mathcal{F}_{H_{1}}} \otimes I_{\mathcal{F}_{H_{2}}} \otimes \cdots, \\
\hat{X}_{n}(t)=I_{\mathcal{F}_{R S W N}} \otimes I_{\mathcal{F}_{H_{0}}} \otimes \cdots \otimes I_{\mathcal{F}_{H_{n-1}}} \otimes X_{n}(t) \otimes I_{\mathcal{F}_{H_{n+1}}} \otimes \cdots .
\end{gathered}
$$

The family $X=\{X(t) / t \geq 0\}$ of self-adjoint operators on $\mathcal{F}_{\mathcal{S}}(\lambda)$ corresponds to a classical stochastic process. For each $t \geq 0$ we will compute the characteristic function $\left\langle\Phi, e^{i s X(t)} \Phi\right\rangle$ of $X(t), s \in \mathbb{R}$. 
Theorem 8.1. Let $\lambda \in(-2,2)$. For all all $t \geq 0$ and $s \in \mathbb{R}$

$$
\left\langle\Phi, e^{i s X(t)} \Phi\right\rangle=(\sec (i s))^{\frac{t}{2}} \exp \left(-\frac{s^{2} t}{8-2 \lambda^{2}}\right) ;
$$

i.e. $X$ can be viewed as the sum of two independent random processes, a continuous binomial/Beta random process (see [3]) and a scaled Brownian motion.

Proof. We know (see [3]) that

$$
\left\langle\Phi_{R S W N}, e^{i s C(t)} \Phi_{R S W N}\right\rangle=(\sec (i s))^{\nu([0, t])}=(\sec (i s))^{\frac{t}{2}}
$$

and

$$
\left\langle\Phi_{n}, e^{i s X_{n}(t)} \Phi_{n}\right\rangle=\exp \left(-\frac{s^{2} \mu_{n}\left(\chi_{[0, t]}\right)}{2}\right)=\exp \left(-\frac{s^{2} t}{8}\left(\frac{\lambda^{2}}{4}\right)^{n}\right) .
$$

Therefore

$$
\begin{aligned}
\left\langle\Phi, e^{i s X(t)} \Phi\right\rangle & =\left\langle\Phi, e^{i s\left(\hat{C}(t)+\sum_{n=0}^{\infty} \hat{X}_{n}(t)\right)} \Phi\right\rangle \\
& =\left\langle\Phi_{R S W N}, e^{i s C(t)} \Phi_{R S W N}\right\rangle \prod_{n=0}^{\infty}\left\langle\Phi_{n}, e^{i s X_{n}(t)} \Phi_{n}\right\rangle \\
& =(\sec (i s))^{\frac{t}{2}} \prod_{n=0}^{\infty} \exp \left(-\frac{s^{2} t}{8}\left(\frac{\lambda^{2}}{4}\right)^{n}\right) \\
& =(\sec (i s))^{\frac{t}{2}} \exp \left(-\frac{s^{2} t}{8} \sum_{n=0}^{\infty}\left(\frac{\lambda^{2}}{4}\right)^{n}\right) \\
& =(\sec (i s))^{\frac{t}{2}} \exp \left(-\frac{s^{2} t}{8} \frac{1}{1-\frac{\lambda^{2}}{4}}\right) \\
& =(\sec (i s))^{\frac{t}{2}} \exp \left(-\frac{s^{2} t}{8-2 \lambda^{2}}\right) .
\end{aligned}
$$

\section{Concluding Remarks}

We have decribed the connection between the impossibility of a joint Fock representation for first and second order white noise and the non-positive definiteness of the Schrödinger Fock kernel of Feinsilver, Kocik and Schott for sets of arbitrarily small measure. We have also described how the positive definite reduction of the Schrödinger Fock kernel, through a restriction of the exponential vectors, leads to a Fock kernel that corresponds to the tensor product of an $s l(2)$ and a Heisenberg Fock space. By computing their characteristic function we identified certain random processes living on the tensor product Fock space. The extension of our study to a full 4-dimensional space-time setting as well as the study of the Schrödinger algebra with the full (three dimensional) rotation group are of future interest.

\section{REFERENCES}

1. L. Accardi, A. Boukas, Renormalized higher powers of white noise (RHPWN) and conformal field theory, Infinite Dimensional Analysis, Quantum Probability, and Related Topics, 9, No. 3 (2006) 353-360. MR2256498(2008e:81069)

2. - The emergence of the Virasoro and $w_{\infty}$ Lie algebras through the renormalized higher powers of quantum white noise, International Journal of Mathematics and Computer Science, 1, No. 3 (2006) 315-342. MR2268746(2008h:81090) 
3. _ Fock representation of the renormalized higher powers of white noise and the Virasoro-Zamolodchikov- $w_{\infty} *-L i e$ algebra, J. Phys. A Math. Theor., 41 (2008). MR 2425825 (2010i:81241)

4. _ Quantum probability, renormalization and infinite dimensional *-Lie algebras, SIGMA (Symmetry, Integrability and Geometry Methods and Applications), 5 (2009), paper 056, 31 pages. MR2506156

5. __ Random variables and positive definite kernels associated with the Schroedinger algebra, Proceedings of the VIII International Workshop "Lie Theory and its Applications in Physics", Varna, Bulgaria, June 16-21, 2009, pages 126-137, American Institute of Physics, AIP Conference Proceedings 1243.

6. L. Accardi, A. Boukas, U. Franz, Renormalized powers of quantum white noise, Infinite Dimensional Analysis, Quantum Probability, and Related Topics, 9, No. 1 (2006) 129-147. MR.2214505 (2007g:60075)

7. L. Accardi , Y. G. Lu, I. V. Volovich, White noise approach to classical and quantum stochastic calculi, Lecture Notes of the Volterra International School of the same title, Trento, Italy (1999), Volterra Center preprint 375, Università di Roma Tor Vergata.

8. P. J. Feinsilver, R. Schott, Algebraic structures and operator calculus. Volumes I and III, Kluwer, 1993. MR.1227095 (94j:22024) MR.1419315 (98k:22001)

9. P. J. Feinsilver, J. Kocik, R. Schott, Representations of the Schroedinger algebra and Appell systems, Fortschr. Phys. 52 (2004), no. 4, 343-359. MR2045066 (2005a:81086)

10. _ Berezin quantization of the Schrödinger algebra, Infinite Dimensional Analysis, Quantum Probability, and Related Topics, 6, No. 1 (2003) 57-71. MR1976870 (2004f:17043)

Centro Vito Volterra, Università di Roma Tor Vergata, via Columbia 2, 00133 Roma, ITALY

E-mail address: accardi@volterra.mat.uniroma2.it

Department of Mathematics, The American College of Greece, Aghia Paraskevi, Athens 15342, GREece

E-mail address: andreasboukas@acg.edu 